\title{
Cyber(r)evolution of the healthcare system: a televisit during the epidemic crisis
}

\section{Marek Krzystanek}

Department and Clinic of Psychiatric Rehabilitation, Department of Psychiatry and Psychotherapy, Faculty of Medical Sciences, Medical University of Silesia in Katowice, Poland

\section{Introduction}

The healthcare system in many countries has been paralyzed due to the coronavirus epidemic. Restrictions on the availability of a specialist doctor and general practitioner may significantly endanger the patient's health, exposing him to the inability to obtain medical consultation and prescribe medication. During this time of epidemic crisis, telemedicine can save the outpatient healthcare system.

Telemedicine involves the use of modern digital technology to remotely provide medical services in the virtual Internet space, using internet platforms and through applications installed on desktops/laptops, tablets and smartphones, or directly from web browsers on websites. Cybermedicine is a more modern term for telemedicine. Cybermedicine is becoming distinct from telemedicine, which also included giving advice over the phone. Cyberpsychiatry is therefore the name of second-generation telemedicine - digital age telemedicine [1].

Numerous publications and reports indicate the possibility of conducting remote therapy. In psychiatry it is effective and safe even in the most severe mental illnesses [2]. In the field of mental health, full equivalence of psychotherapy conducted in telemedicine and analogue form has also been demonstrated [3].

Doctors need clear guidelines on how to conduct such a visit. A televisit is a form of remote treatment that can be carried out by a doctor of any specialty. In specialties such as psychiatry, in which the main source of information about the patient's health is an interview, televisits can even constitute the majority of visits. In other fields of medicine, especially surgical ones, telemedicine may be supported by telemetry and telerobotics - but these areas of medicine still need to be developed so that they could support or even replace the work of a doctor.
The present time can cause a rapid and dynamic increase in interest in cybermedicine and cause dynamic growth in the mobile application market. A time of epidemic crisis, creating the necessity of making televisits between a doctor and a patient, will also cause a revolution in the thinking of many patients. If there is no other way to contact a doctor other than remote contact, the barrier of technophobia - fear of new technology - common especially in older people, will be overcome.

Scientific bodies around the world should now develop regulations and guidelines for conducting televisits. I wanted to familiarize readers with the guidelines that were formulated in Poland by the Scientific Section of Telepsychiatry of the Polish Psychiatric Association, of which I am chairman [4]. These guidelines can be an important voice in the discussion aimed at promoting the provision of remote medical services now.

\section{Polish recommendations for conducting televisits}

A remote visit (televisit) should be treated as fully equivalent to an analogue visit, carried out in a clinic or private doctor's office. Under normal circumstances, analogue visits and remote visits may be alternated and complement each other. Of course, the doctor keeps the patient's medical records on these visits. In Poland, the televisit is issued by the National Health Fund (NHF), which allows the medical financial contract to be carried out in the form of remote treatment. With the current development of telemetry, a televisit does not yet allow a physical examination of the patient; therefore the first visit should be a visit to a clinic or private doctor's office. However, at the present critical time, when access to a doctor is limited, exceptionally even the first visit can take the form of a video-visit, and the patient's physical examination should be completed after the restrictions 
preventing the spread of coronavirus infection have been withdrawn.

A televisit should be conducted using an audio and video channel. This allows one to create an empathic relationship between the patient and the doctor. This combination also allows more reliable and appropriate assessment of the patient, his facial expressions and gestures, as well as subtle nuances in his behavior during the conversation. If technical difficulties prevent such contact, the doctor should strive for the patient to use another person's smartphone next time or to install applications on his smartphone that enable the use of a video connection.

An important problem that arises during a televisit is the identification of a patient who the doctor is seeing for the first time in his life. In Poland, we recommend that the patient show any ID with his photo to the smartphone camera. However, this does not guarantee the authenticity of the identity card shown. There is currently no effective procedure in Poland that gives 100\% certainty to determine the patient's identity, which is why, in case of any suspicions, the doctor may not provide the patient with an e-prescription code, but rather recommend opening the NHF website and acquiring the code, possible only through a trusted signature.

During the televisit, the patient should ensure intimate conditions in the place where he is talking. He should be alone in this room, and if there are other people in the house, he can use headphones with a microphone instead of speakers so that only the patient can hear the doctor's questions. If the patient wishes another person to be present during the televisit, it should always be his decision. This can be especially helpful for older people, when the other person can help them connect or solve any possible technical problems.

Before the televisit, the patient, in addition to the identity card, should prepare his medical data, data on subsequent medical treatment, lab test results, statements from other doctors and a list of medications he had used before and now. The doctor may ask the patient to send scans of these medical documents (or legible pictures from a mobile phone) to the email address provided by him.

At the time of the televisit, the doctor may prescribe the patient an e-prescription, and issue an electronic sick leave. Unfortunately, it is not yet possible to write electronic referrals to a psychiatric hospital in Poland, which the author advocates. In the case of a health certificate in Poland, it must be signed and sealed with a doctor's stamp. In such a situation, if the patient needs such a document, we send him a scan and a traditional letter.

Concerning legal issues, if during the televisit the doctor realizes that the patient's state of health requires a stay in the hospital, he should refer the patient to the hospital emergency department. Importantly, the physician is not responsible for incomplete, false or incorrect information being provided by the patient, especially in the case of third party data being provided without their knowledge or consent. The consequences of providing incorrect, incomplete, false, misleading or otherwise incorrect data are the sole responsibility of the patient.

\section{Conclusions}

Nowadays, during the televisit the doctor should also discuss with the patient recommendations regarding the use of personal protective equipment (e.g. frequent hand washing, sneezing or coughing into a clenched fist or elbow) and behaviors protecting against accidental infection (i.e. limiting leaving the house and direct contact with others, maintaining a distance of at least $2 \mathrm{~m}$ when dealing with people outside the home).

The physician should calm down the patient who may have reactive emotional disturbances associated with the epidemic threat. It is necessary to find out whether the patient has suffered from media misinformation, present facts and make sure the patient has accurate knowledge. Such medical education may be enough to reassure patients. The physician should also discuss with the patient what to do in the case of contact with a person infected with SARS-CoV-2 and what to do if they suspect that they have symptoms of COVID-19 disease.

During the epidemic crisis, the televisits should also include issues related to hygiene of life and hygiene of sleep. The patient should hear about the need for calmness, relaxation (including recommendations for breathing exercises or relaxation training on-line, e.g. on youtube.com), conversations with loved ones allowing them to relieve tension, maintaining a regular rhythm of life, avoiding stimulants or psychoactive substances as a male habit strategy of regulation of emotions, pursuing hobbies and interests, regular meals and sleep hygiene. These soft recommendations can help the patient maintain good health.

Televisits can help patients survive the time of epidemic crisis. It is possible that the world of healthcare after the coronavirus epidemic will appear different than before. Within a few months, perception of telemedicine/cybermedicine will change and the frequency of using telemedicine applications may significantly increase. Such a change may be permanent and telemedicine will become a permanent element of the healthcare system.

The author declares no conflict of interest. 


\section{References}

1. Krzystanek M, Domagała B. Cyberpsychiatria-leczenie w cyberprzestrzeni Internetu [Cyberpsychiatry - a treatment in cyberspace of Internet]. Psychiatr Dypl 2019; 16: 15-20.

2. Krzystanek M, Borkowski M, Skałacka K, Krysta K. A telemedicine platform to improve clinical parameters in paranoid schizophrenia patients: Results of a one-year randomized study. Schizophr Res 2019; 204: 389-396, DOI: 10.1016/j. schres.2018.08.016.
3. Krzystanek M, Stolarczyk A, Borkowski M, Murawiec S. Telemedyczna terapia poznawczo-behawioralna [Telemedical cognitive-behavioral therapy]. Psychiatria 2018; 15: 110-116.

4. https://psychiatria.org.pl/aktualnosci,tekst,392,rekomendacje. 